\title{
Closure of a persistent esophagopleural fistula using an over-the-scope clipping device
}

Esophagopleural fistula (EPF) is considered to be a rare condition, usually occurring after pneumonectomy. When it does occur, it is accompanied by significant morbidity and the associated mortality is high [1].

We describe the case of a 44-year-old man with chronic active hepatitis B complicated by cirrhosis. While awaiting orthotopic liver transplantation, the patient had an esophageal variceal hemorrhage and underwent several sessions of variceal sclerotherapy. Orthotopic liver transplantation 2 months after the sclerotherapy treatment was successful, but a further 2 months later the patient developed a refractory empyema with continuous pleural drainage. Pulmonary malignancy and infectious causes were ruled out by percutaneous drainage with cytologic evaluation and culture of the pleural effusion. Cultures and polymerase chain reaction assay for tuberculosis in the pleural fluid were also negative. Chest CT suggested the presence of an EPF ( $\bullet$ Fig. 1). Upper endoscopy was performed for further evaluation and revealed a $5-\mathrm{mm}$ fistula orifice in the mid-esophagus. The esophageal mucosa surrounding the fistula appeared scarred and fibrotic.

After consultation with the transplant surgical service, we performed upper endoscopy and used a single $10-\mathrm{mm}$ over-thescope clip (OTSC; Ovesco Endoscopy AG, Tübingen, Germany) to close the fistula ( Fig. 2). The clip was of the "atraumatic type" with blunt teeth ( $\bullet$ Fig.3). The fistula was closed with complete cessation of pleural drainage. Insertion of a removable esophageal stent was not considered due to the expected high risk of stent migration, and 2 weeks after the OTSC placement, the pleural drainage recurred. Chest radiography showed no evidence of the presence of a clip in the mid-esophagus, so a second therapeutic upper endoscopy was performed and an Ovesco $10-\mathrm{mm}$ "spiked-teeth" clip was placed, successfully and permanently closing the EPF ( Fig.4), with resolution of the empyema. Barium esophagogram 3 months later was normal without any evidence of clip or extravasation of contrast. The patient continues to do well now, 5 months after closure of the fistula.

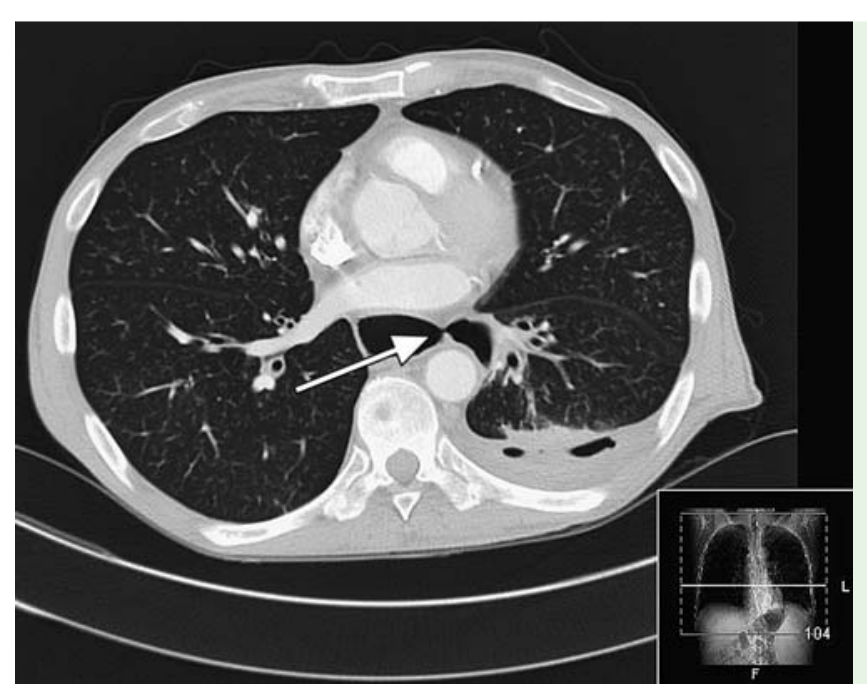

Fig. 1 Computed tomogram showing esophagopleural fistula in a 44-year-old man.

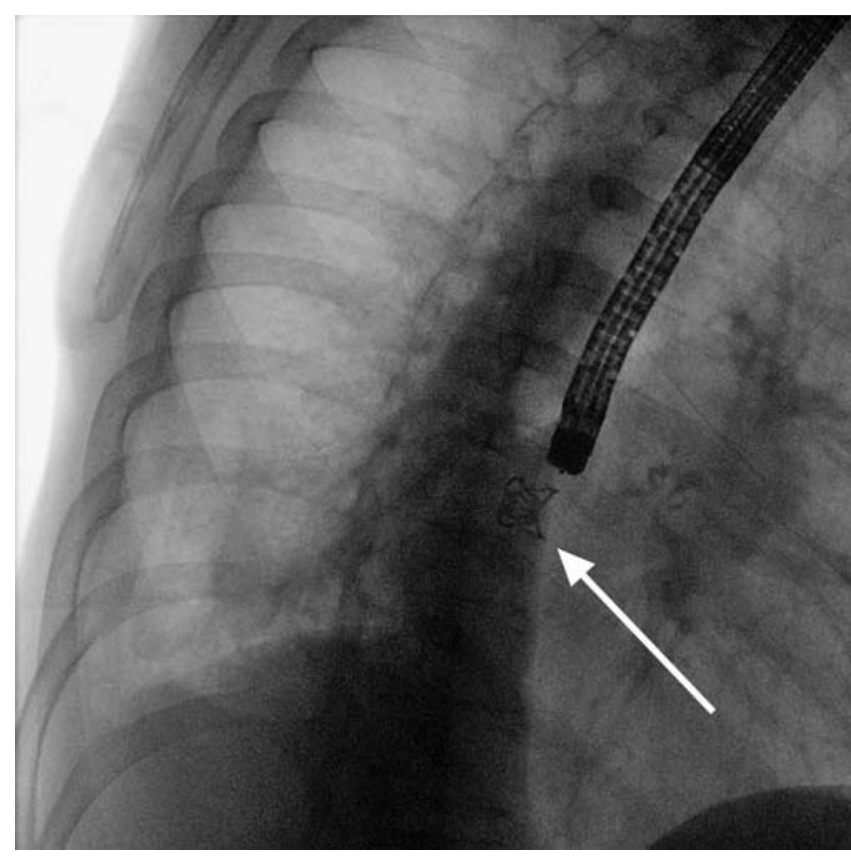

Fig. 2 Over-the-scope deployment of a clip.

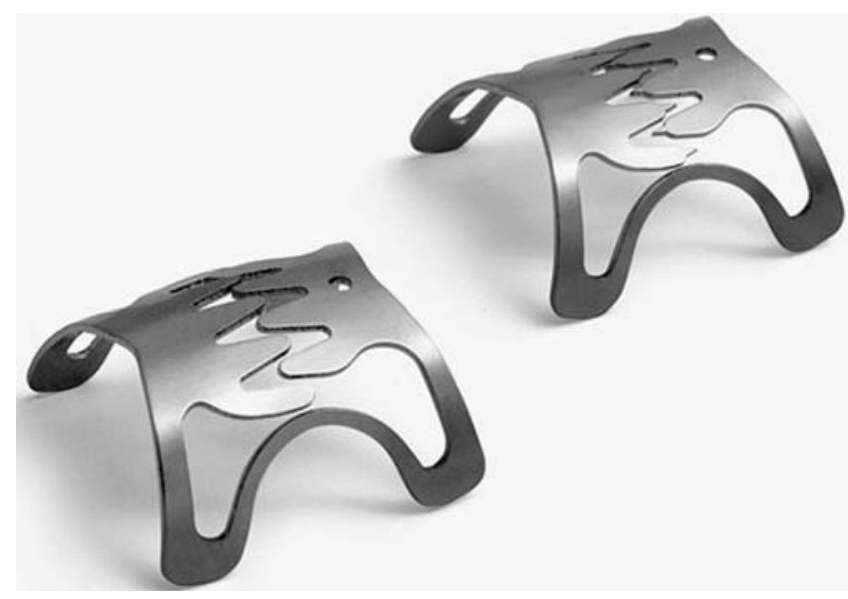

Fig. 3 "Atraumatic" type of over-the-scope clip, with blunt teeth. 


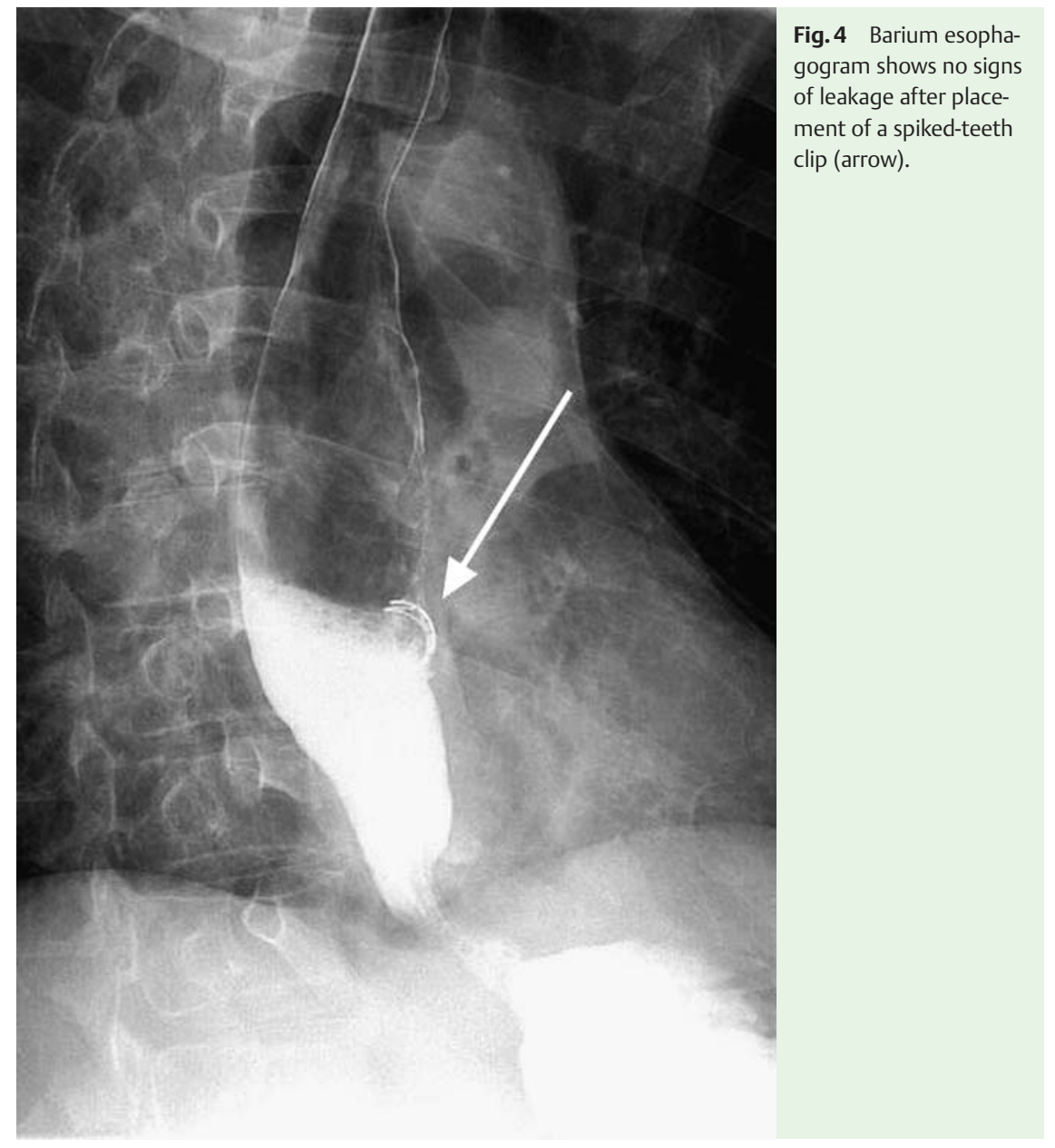

EPF is considered to be a rare but often fatal complication. The vast majority of EPFs develop after pneumonectomy. To the best of our knowledge, only three cases of post-sclerotherapy EPF have been reported; all were pediatric cases in which the EPF was treated surgically [24]. Small pleural effusions have been reported in up to $50 \%$ of patients who have undergone esophageal variceal sclerotherapy [5]. As perforation with fistula formation is a consequence of chemical transmural necrosis rather than mechanical injury, the manifestation of EPF is often delayed.

Only a single report has described endoscopic management of EPF, and in that case the endoscopic management was

\section{Khamaysi ${ }^{1,2}$, A. Suissa ${ }^{1,2}$, K. Yassin ${ }^{1,2}$,} I. M. Gralnek ${ }^{1,2}$

${ }^{1}$ Department of Gastroenterology and Interventional Endoscopy Unit, Rambam Health Care Campus, Haifa, Israel

${ }^{2}$ Rappaport Faculty of Medicine, TechnionIsrael Institute of Technology, Haifa, Israel

\section{References}

1 Wechsler RJ. CT of esophageal-pleural fistulae. AJR Am J Roentgenol 1986; 147: $907-$ 909

2 Prasad H, Poddar U, Thapa BR et al. Esophagopleural fistula after endoscopic sclerotherapy in a child. Gastrointest Endosc 2000; 52: 804-806

3 Mabrut JY, Druez P, Goncette L et al. Drainage-lavage and closure of a late esophageal perforation with esophagopleural fistula and encysted pleural effusion after endoscopic injection sclerotherapy for varices [in French]. Gastroenterol Clin Biol 2003; 27: $1031-1034$

4 Chertow GM, Marcantonio ER, Wells RG. Saccharomyces cerevisiae empyema in a patient with esophago-pleural fistula complicating variceal sclerotherapy. Chest 1991; 99: 1518-1519

5 Edling JE, Bacon BR. Pleuropulmonary complications of endoscopic variceal sclerotherapy. Chest 1991; 99: 1252 - 1257

6 Bonin EA, Wong Kee Song LM, Gostout ZS et al. Closure of a persistent esophagopleural fistula assisted by a novel endoscopic suturing system. Endoscopy (UCTN) 2012; 44: E8-E9

\section{Bibliography}

Dol http://dx.doi.org/

$10.1055 / \mathrm{s}-0032-1325860$

Endoscopy 2013; 45: E29-E30

(c) Georg Thieme Verlag KG

Stuttgart · New York

ISSN 0013-726X

first clip was of the blunt-teeth type so the margins of the fistula could not be grasped tightly. The second clip was of the spiked-jaws type, providing an improved ability to grasp the fibrotic esophageal mucosa. In the future, use of a large over-the-scope clipping device should be considered in cases of persistent EPF.

Endoscopy_UCTN_Code_TTT_1AO_2AI

Competing interests: None

\section{Corresponding author}

\section{Khamaysi, MD}

Department of Gastroenterology and

Interventional Endoscopy Unit

Rambam Health Care Campus

POB 9602

Haifa 31096

Israel

Fax: +972-4-8543058

k_iyad@rambam.health.gov.il 